\title{
Strong linearly independent vectors in semilinear spaces and their applications
}

\author{
Qian-yu Shu ${ }^{1}$ Qing-quan Xiong ${ }^{2}$
}

College of Mathematics and Software Science, Sichuan Normal University,Chengdu, Sichuan 610066, P. R. China

\begin{abstract}
The aim of this contribution is to discuss the characterizations of $\mathcal{L}$-semilinear spaces which are generated by strong linearly independent vectors. First, we show that the basis in $\mathcal{L}$-semilinear spaces which are generated by strong linearly independent vectors is also strong linearly independent. Then we prove that the analogue of the Kronecker-Capelli theorem is valid for systems of equations.
\end{abstract}

Keywords: Semilinear spaces; Strong linearly independent; Basis;Kronecker-Capelli theorem

\section{Introduction}

The study of semilinear structures over zerosumfree semirings has a long history. In 1979, CuninghameGreen built a theory similar to that of linear algebra in min-plus algebra, for instance in [5], systems of linear equations, eigenvalue problems, independence, rank and dimension. Since then, a number of works on semilinear structure over zerosumfree semirings were published (see e.g. [2, 3, 4, 10, 11]). In 2007, Di Nola et al. used the notions of semirings and semimodule to introduce the concept of semilinear space in the MV-algebraic setting, and obtained some similar results as those of classical linear algebras (see [7]). In 2010, Perfilieva and Kupka showed that the necessary condition of the Kronecker-Capelli theorem is valid for systems of equations in a semilinear space of $n$-dimensional vectors (see [12]), Zhao and Wang gave a sufficient condition that each basis in similinear spaces of $n$ dimensional vectors has the same number of elements over commutative zerosumfree semirings (see [20]), moreover, $1 n$ 2011, they obtained a necessary and sufficient condition that each basis has the same number of elements over join-semirings (see [21]), where a join-semiring is just a kind of zerosumfree semiring. In 2011, Shu and Wang showed some necessary and sufficient conditions that each basis has the same number of elements over commutative zerosumfree semirings and proved that a set of vectors is a basis if and only if they are standard orthogonal (see [15]). In 2012, Shu and Wang showed that a set of linearly independent non standard orthogonal vectors can not be orthogonalized if it has at least two nonzero vectors, and proved that the analog of the Kronecker-Capelli theorem was valid for systems of equations when the column vectors of coefficient matrix was standard orthogonal(see [16]). It is obvious that the problem is still open if the column vectors of coefficient matrix is not standard orthogonal. In this paper, we shall investigate it when the column vectors of coefficient matrix is strong linearly independent. First, we will show that the basis in $\mathcal{L}$-semilinear spaces which are generated by strong linearly independent vectors is also strong linearly independent. Then we prove that the analogue of the Kronecker-Capelli theorem is valid for systems of equations.

\section{Semilinear Spaces}

In this section, we give some definitions and preliminary lemmas.

Definition 2.1 ( Golan [8],Zimmermannn[22]) A semiring $\mathcal{L}=\langle L,+, \cdot, 0,1\rangle$ is an algebraic structure with the following properties:

(i) $(L,+, 0)$ is a commutative monoid,

(ii) $(L, \cdot, 1)$ is a monoid,

(iii) $r \cdot(s+t)=r \cdot s+r \cdot t$ and $(s+t) \cdot r=s \cdot r+t \cdot r$ hold for all $r, s, t \in L$,

(iv) $0 \cdot r=r \cdot 0=0$ holds for all $r \in L$,

(v) $0 \neq 1$.

A semiring $\mathcal{L}$ is commutative if $r \cdot r^{\prime}=r^{\prime} \cdot r$ for all $r, r^{\prime} \in L$. A semiring $\mathcal{L}$ is called zerosumfree if $a+b=0$ implies that $a=b=0$ for any $a, b \in L$.

Example 2.1 $\mathcal{L}=\langle\mathbb{R} \cup\{-\infty\},+, \cdot,-\infty, 0\rangle$ is a semiring, where $a+b=\max \{a, b\}$ and $a \cdot b=a+b$ for $a, b \in \mathbb{R} \cup\{-\infty\}$ in which the last + stands for the usual addition of real numbers.

Note that the semiring $\mathcal{L}=\langle\mathbb{R} \cup$ $\{-\infty\},+, \cdot,-\infty, 0\rangle$ is usually called a max-plus algebra or a schedule algebra (see e.g. $[1,2,6]$ ).

Example 2.2 It is clear that $\mathbb{N}$ with the usual operations of addition and multiplication of integers is a commutative zerosumfree semiring.

Example 2.3 (Zhao and Wang [20]) The following are examples of commutative zerosumfree semirings:

(i) The real interval $[0,1]$ under the operations $a+b=\max \{a, b\}$ and $a \cdot b=\min \{a, b\}$ for all $a, b \in[0,1]$;

(ii) The nonnegative real numbers with the usual operations of addition and multiplication; 
(iii) The nonnegative integers under the operations $a+b=$ g.c.d. $\{a, b\}$ and $a \cdot b=$ l.c.m. $\{a, b\}$ for nonnegative integers $a$ and $b$, and g.c.d. (resp. l.c.m.) stands for the greatest (resp. least) common divisor (resp. multiple) between $a$ and $b$.

\section{Definition 2.2 (Zimmermannn[22]) Let}

$\mathcal{L}=\langle L,+, \cdot, 0,1\rangle$ be a semiring and let $\mathcal{A}=\left\langle A,+_{A}, 0_{A}\right\rangle$ be a commutative monoid. If ${ }^{*}: L \times A \rightarrow A$ is an external multiplication such that

(i) $\left(r \cdot r^{\prime}\right) * a=r *\left(r^{\prime} * a\right)$,

(ii) $r *\left(a+{ }_{A} a^{\prime}\right)=r * a+{ }_{A} r * a^{\prime}$,

(iii) $\left(r+r^{\prime}\right) * a=r * a+{ }_{A} r^{\prime} * a$,

(iv) $1 * a=a$,

(v) $0 * a=r * 0_{A}=0_{A}$

for all $r, r^{\prime} \in L$ and $a, a^{\prime} \in A$ then $\left\langle\mathcal{L},+, \cdot, 0,1 ; * ; A,+_{A}, 0_{A}\right\rangle$ is called a left $\mathcal{L}$ semimodule. The definition of right $\mathcal{L}$-semimodule is analogous, where the external multiplication is defined as a function $A \times L \rightarrow A$.

The following definition is a general version of that of a semilinear space in [7]:

Definition 2.3 Let $\mathcal{L}=\langle L,+, \cdot, 0,1\rangle$ be a semiring. Then a semimodule over $\mathcal{L}$ is called an $\mathcal{L}$ semilinear space.

Note that in Definition 2.3, a semimodule stands for a left $\mathcal{L}$-semimodule or a right $\mathcal{L}$-semimodule as in [7]. Elements of an $\mathcal{L}$-semilinear space will be called vectors and elements of a semiring scalars. The former will be denoted by bold letters to distinguish them from scalars.

Without loss of generality, in what follows, we consider left $\mathcal{L}$-semimodules for convenience of notation. Let $\underline{n}=\{1, \cdots, n\}$. Then we can construct an $\mathcal{L}$-semilinear space as follows.

Definition 2.4 (Di Nola et al.[7]) Let $\left\langle L,+, \cdot, 0,1 ; * ; A,+_{A}, 0_{A}\right\rangle$ be an $\mathcal{L}$-semilinear space. The expression

$$
\lambda_{1} \mathbf{a}_{1}+{ }_{A} \cdots+_{A} \lambda_{n} \mathbf{a}_{n},
$$

where $\lambda_{1}, \cdots, \lambda_{n} \in L$ are scalars is called a linear combination of vectors $\mathbf{a}_{1}, \cdots, \mathbf{a}_{n} \in A$.

Example 2.4 (Shu and Wang, 2011) Let $\mathcal{L}=\langle L,+, \cdot, 0,1\rangle$ be a semiring. For each $n \geqslant 1$, let

$$
V_{n}(L)=\left\{\left(a_{1}, a_{2}, \cdots, a_{n}\right)^{T}: a_{i} \in L, i \in \underline{n}\right\} .
$$

Define

$$
\begin{gathered}
\mathbf{x}+\mathbf{y}=\left(x_{1}+y_{1}, x_{2}+y_{2}, \cdots, x_{n}+y_{n}\right)^{T}, \\
r * \mathbf{x}=\left(r \cdot x_{1}, r \cdot x_{2}, \cdots, r \cdot x_{n}\right)^{T}
\end{gathered}
$$

for all $\mathbf{x}=\left(x_{1}, x_{2}, \cdots, x_{n}\right)^{T}, \mathbf{y}=$ $\left(y_{1}, y_{2}, \cdots, y_{n}\right)^{T} \in V_{n}(L)$ and $r \in L$, where $\left(x_{1}, x_{2}, \cdots, x_{n}\right)^{T}$ denotes the transpose of $\left(x_{1}, x_{2}, \cdots, x_{n}\right)$. Then $\mathcal{V}_{n}=$ $\left\langle L,+, \cdot, 0,1 ; * ; V_{n}(L),+, \mathbf{0}_{n \times 1}\right\rangle$ is an $\mathcal{L}$-semilinear space with $\mathbf{0}_{n \times 1}=(0,0, \cdots, 0)^{T}$.
From now on, without causing confusion we use $r \mathbf{a}$ instead of $r * \mathbf{a}$ for all $r \in L$ and $\mathbf{a} \in A$ in an $\mathcal{L}$-semilinear space $\left\langle L,+, \cdot, 0,1 ; * ; A,+_{A}, 0_{A}\right\rangle$.

Definition 2.5 (Di Nola et al., 2007) In $\mathcal{L}$ semilinear space, a single vector $\mathbf{a}$ is linearly independent. Vectors $\mathbf{a}_{1}, \cdots, \mathbf{a}_{n}, n \geqslant 2$, are linearly independent if none of them can be represented by a linear combination of the others. Otherwise, we say that vectors $\mathbf{a}_{1}, \cdots, \mathbf{a}_{n}$ are linearly dependent. An infinite set of vectors is linearly independent if any finite subset of it is linearly independent.

Remark 2.1 In general, the cardinality of a basis is not unique (see e.g. [7, 15, 20, 21]).

We denote by $M_{m \times n}(L)$ the set of all $m \times n$ matrices over a semiring $\mathcal{L}=\langle L,+, \cdot, 0,1\rangle$. Especially let $M_{n}(L)=M_{n \times n}(L)$. Given $A=\left(a_{i j}\right)_{m \times n}, B=$ $\left(b_{i j}\right)_{m \times n} \in M_{m \times n}(L)$ and $C=\left(c_{i j}\right)_{n \times l} \in M_{n \times l}(L)$, we define that

$$
\begin{gathered}
A+B=\left(a_{i j}+b_{i j}\right)_{m \times n}, \\
A C=\left(\sum_{k \in \underline{n}} a_{i k} \cdot c_{k j}\right)_{m \times l}, \\
\lambda A=\left(\lambda a_{i j}\right)_{m \times n} \text { for all } \lambda \in L .
\end{gathered}
$$

Then $\left\langle M_{n}(L),+, \cdot, O_{n}, I_{n}\right\rangle$ is a semiring with

$O_{n}=\left(\begin{array}{cccc}0 & 0 & \cdots & 0 \\ 0 & 0 & \cdots & 0 \\ & \cdots & \cdots & \\ 0 & 0 & \cdots & 0\end{array}\right)$ and $I_{n}=\left(\begin{array}{cccc}1 & 0 & \cdots & 0 \\ 0 & 1 & \cdots & 0 \\ & \cdots & \cdots & \\ 0 & 0 & \cdots & 1\end{array}\right)$

A nonempty subset $G$ of an $\mathcal{L}$-semilinear space is called a set of generators if every element of the $\mathcal{L}$ semilinear space is a linear combination of elements in $G$ (see [6]). Let $S$ be a set of generators of $\mathcal{L}$ semilinear space $\mathcal{A}$. Then denote as $\mathcal{A}=\langle S\rangle$.

Definition 2.6 (Golan, 1999) A linearly independent set of generators of an $\mathcal{L}$-semilinear space $\mathcal{A}$ is called a basis of $\mathcal{A}$.

Definition 2.7 (Golan, 1999) An element $a \in$ $L$ is called invertible in a semiring $\mathcal{L}$ if there exists an element $b \in L$ such that $a b=b a=1$. Such element $b$ is called an inverse of $a$, it is clear that the inverse of $a$ is unique, then denote it by $a^{-1}$. Let $U(L)$ denote the set of all invertible elements in a semiring $\mathcal{L}$.

Definition 2.8 (Wang and Shu, 2014) In $\mathcal{V}_{n}$, vectors $\mathbf{a}_{1}, \cdots, \mathbf{a}_{n}$ are semi-linearly dependent if and only if there exist two nonempty disjoint subsets of indices $J_{1} \subset \underline{n}$ and $J_{2} \subset \underline{n}$ together with $0 \neq \lambda_{i} \in$ $L, i \in J_{1} \cup J_{2}$, such that $\sum_{j \in J_{1}} \lambda_{j} \boldsymbol{a}_{j}=\sum_{j \in J_{2}} \lambda_{j} \boldsymbol{a}_{j}$ with either $\left|J_{1}\right|,\left|J_{2}\right| \geq 2$ or if $\left|J_{i}\right|=1$ for some $i \in\{1,2\}$ then $\lambda_{j} \notin U(L)$ for every $j \in J_{i}$. 
Note that if a set of vectors is semi-linearly dependent, then it is linearly independent. In general, the converse is not true. For example, vectors $\left(\begin{array}{l}1 \\ 0 \\ 0 \\ 0\end{array}\right),\left(\begin{array}{l}0 \\ 1 \\ 0 \\ 0\end{array}\right),\left(\begin{array}{l}0 \\ 0 \\ 1 \\ 0\end{array}\right),\left(\begin{array}{l}0 \\ 0 \\ 0 \\ 1\end{array}\right)$ are linearly independent and not semi-linearly dependent. Some authors defined linear dependence of vectors as that: vectors $\mathbf{a}_{1}, \cdots, \mathbf{a}_{n}, n \geqslant 2$, are linearly dependent if and only if there exist two nonempty disjoint subsets of indices $J_{1} \subset \underline{n}$ and $J_{2} \subset \underline{n}$ together with $0 \neq \lambda_{i} \in L, i \in J_{1} \cup J_{2}$, such that $\sum_{j \in J_{1}} \lambda_{j} \boldsymbol{a}_{j}=$ $\sum_{j \in J_{2}} \lambda_{j} \boldsymbol{a}_{j}$ (see e.g. [8]). This definition means that if a set of vectors is linearly dependent if and only if it is either linearly dependent in the sense of Definition 2.5 or semi-linearly dependent in the sense of Definition 2.8.

Definition 2.9 (Wang and Shu, 2014) In $\mathcal{V}_{n}$, a single vector $\mathbf{a}$ is strong linearly independent. Vectors $\mathbf{a}_{1}, \cdots, \mathbf{a}_{n}, n \geqslant 2$, are strong linearly independent if and only if they are linearly independent and not semi-linearly dependent.

\section{Bases in $\mathcal{L}$-semilinear spaces which are generated by strong linearly independent vectors}

Definitions 2.8 and 2.9 we know that, every set of linearly independent vectors either strong linearly independent or semi-linearly dependent. And on the other hand, it is clear that a standard orthogonal vectors are strong linearly independent, but the converse is not true (see $[17,19])$. We know that in $\mathcal{L}$-semilinear space over commutative semirings which are generated by standard orthogonal, the basis is standard orthogonal, which is different with the conclusion in classical algebra. A natural question to ask now is: if the set of generators of an $\mathcal{L}$-semilinear space is strong linearly independent, what about the other bases? In this section, we shall solve this problem under certain condition. In what follows, we always suppose that $\mathcal{L}=\langle L,+, \cdot, 0,1\rangle$ is a commutative zerosumfree semiring.

Definition 3.1 (Golan, 1999) An element $a$ in a semiring $\mathcal{L}$ is said to be cancellable if and only if $a+b=a+c$ implies $b=c$ for every $b, c \in L$. We denote the set of all cancellable elements of $L$ by $K^{+}(L)$.

We now present another weak version of the condition of having an additive inverse. Set $W(L)=$ $\{a \in L$ : for every $b \in L$ there exists an element $r$ of $L$ such that either $a+r=b$ or $a=b+r\}$. Clearly $W(L)$ is nonempty since $V(L) \subseteq W(L)$. If $W(L)=L$ then the semiring $\mathcal{L}$ is said to be yoked (see $[8])$.

Definition 3.2 (Golan [8]) A nonzero element $a$ of a semiring $\mathcal{L}$ is a left zero divisor if and only if there exists a nonzero element $b$ of $L$ satisfying $a b=0$. It is a right zero divisor if and only if there exists a nonzero element $b$ of $L$ satisfying $b a=0$. It is a zero divisor if and only if it is both a left and a right zero divisor. A semiring $\mathcal{L}$ having no zero divisor is said to be entire.

Definition 3.3 (Kuntzman, 1972) Let

$A \in M_{n}(L)$. Denote $P$ (resp. $Q$ ) the set of even (resp. odd) permutations of the set $\underline{n}$. A bideterminant $\operatorname{det}(A)$ of $A$ is an ordered pair

$$
\operatorname{det}(A)=\left(\operatorname{det}_{1}(A), \operatorname{det}_{2}(A)\right)
$$

such that $\operatorname{det}_{1}(A), \operatorname{det}_{2}(A) \in L$ with

$$
\operatorname{det}_{1}(A)=\sum_{\sigma \in P} a_{1, \sigma(1)} a_{2, \sigma(2)} \cdots a_{n, \sigma(n)}
$$

and

$$
\operatorname{det}_{2}(A)=\sum_{\sigma \in Q} a_{1, \sigma(1)} a_{2, \sigma(2)} \cdots a_{n, \sigma(n)} .
$$

Note that if $\operatorname{det}_{1}(A)=\operatorname{det}_{2}(A)$, then $\operatorname{det}(A) \equiv 0$ (see [19]). Otherwise, we use symbols $\operatorname{det}(A) \not \equiv 0$.

Lemma 3.1 (Shu and Wang, 2013) In a cancellative, yoked and entire semiring $\mathcal{L}$, let $\mathcal{W}=$ $\left\langle\mathbf{x}_{1}, \mathbf{x}_{2}, \cdots, \mathbf{x}_{t}\right\rangle$, where $\mathbf{x}_{1}, \mathbf{x}_{2}, \cdots, \mathbf{x}_{t}$ are strong linearly independent in $\mathcal{V}_{n}$. Then every a $\in \mathcal{W}$, can be uniquely represented by a linear combination of $\mathbf{x}_{1}, \mathbf{x}_{2}, \cdots, \mathbf{x}_{t}$.

Lemma 3.2 (Poplin et al., 2004) If $A, B \quad \in$ $M_{n}(L)$, then

$$
\begin{aligned}
& \operatorname{det}_{1}(A B)+\operatorname{det}_{1}(A) \operatorname{det}_{2}(B)+\operatorname{det}_{2}(A) \operatorname{det}_{1}(B) \\
= & \operatorname{det}_{2}(A B)+\operatorname{det}_{1}(A) \operatorname{det}_{1}(B)+\operatorname{det}_{2}(A) \operatorname{det}_{2}(B) .
\end{aligned}
$$

Lemma 3.3 (Wang and Shu, 2014) In a cancellative, yoked and entire semiring $\mathcal{L}$, let $A=$ $\left(a_{i j}\right) \in M_{n}(L)$. Then $\operatorname{det}(A) \equiv 0$ if and only if the column-vectors of $A$ are linearly dependent or semi-linearly dependent.

Theorem 3.1 In a cancellative, yoked and entire semiring $\mathcal{L}$, let $\mathcal{W}=\left\langle\mathbf{x}_{1}, \mathbf{x}_{2}, \cdots, \mathbf{x}_{s}\right\rangle$ with $\left\{\mathbf{x}_{1}, \mathbf{x}_{2}, \cdots, \mathbf{x}_{s}\right\}$ a set of strong linearly independent vectors in $\mathcal{V}_{n}$. If $\left\{\mathbf{y}_{1}, \mathbf{y}_{2}, \cdots, \mathbf{y}_{p}\right\}$ is a basis of $\mathcal{W}$, then $\mathbf{y}_{1}, \mathbf{y}_{2}, \cdots, \mathbf{y}_{p}$ are strong linearly independent and $s=p$.

Proof. If By Lemmas 3.1 we can prove that $s=p$. Let $\mathbf{y}_{i}=\sum_{j=1}^{s} a_{i j} \mathbf{x}_{j}$ with $a_{i j} \in L$ for any $i \in \underline{s}$. Thus

$$
\left(\mathbf{y}_{1}, \mathbf{y}_{2}, \cdots, \mathbf{y}_{s}\right)=\left(\mathbf{x}_{1}, \mathbf{x}_{2}, \cdots, \mathbf{x}_{s}\right) A
$$

with

$$
A=\left(\begin{array}{ccc}
a_{11} & \cdots & a_{s 1} \\
\cdots & \cdots & \cdots \\
a_{1 s} & \cdots & a_{s s}
\end{array}\right)
$$


Since $\mathbf{y}_{1}, \mathbf{y}_{2}, \cdots, \mathbf{y}_{s}$ are linearly independent, then every column of $A$ has nonzero element. In a similar way, we can let

$$
\left(\mathbf{x}_{1}, \mathbf{x}_{2}, \cdots, \mathbf{x}_{s}\right)=\left(\mathbf{y}_{1}, \mathbf{y}_{2}, \cdots, \mathbf{y}_{s}\right) B
$$

with $B=\left(b_{j i}\right), b_{j i} \in L, i, j \in \underline{s}$, and every row of $B$ has nonzero element. Therefore,

$$
\left(\mathbf{x}_{1}, \mathbf{x}_{2}, \cdots, \mathbf{x}_{s}\right)=\left(\mathbf{x}_{1}, \mathbf{x}_{2}, \cdots, \mathbf{x}_{s}\right) A B .
$$

Since $\left\{\mathbf{y}_{1}, \mathbf{y}_{2}, \cdots, \mathbf{y}_{s}\right\}$ is linearly independent, then by Definitions 2.8 and 2.9, we know that $\left\{\mathbf{y}_{1}, \mathbf{y}_{2}, \cdots, \mathbf{y}_{s}\right\}$ is either strong linearly independent or semi-linearly dependent. If it is semilinearly dependent, then by Definition 2.8 , there exist two disjoint subsets of indices $J_{1} \subset \underline{n}$ and $J_{2} \subset \underline{n}$ together with $0 \neq \lambda_{j} \in L, j \in J_{1} \cup J_{2}$, such that $\sum_{j \in J_{1}} \lambda_{j} \mathbf{y}_{j}=\sum_{j \in J_{2}} \lambda_{j} \mathbf{y}_{j}$ with $\left|J_{1}\right|,\left|J_{2}\right| \geq 2$ or there exists $\left|J_{i}\right|=1, i \in\{1,2\}$ such that if $j \in J_{i}$, then $\lambda_{j} \notin U(L)$. Thus

$$
\sum_{j \in J_{1}} \lambda_{j}\left(\sum_{k=1}^{s} a_{j k} \mathbf{x}_{k}\right)=\sum_{j \in J_{2}} \lambda_{j}\left(\sum_{k=1}^{s} a_{j k} \mathbf{x}_{k}\right),
$$

where $\left|J_{1}\right|,\left|J_{2}\right| \geq 2$ or if $\left|J_{i}\right|=1$ for some $i \in\{1,2\}$ then $\lambda_{j} \notin U(L)$ for every $j \in J_{i}$. In the first case, $\left|J_{1}\right|,\left|J_{2}\right| \geq 2$, then we have

$$
\begin{aligned}
& \sum_{j \in J_{1}} \lambda_{j} a_{j 1} \mathbf{x}_{1}+\cdots+\sum_{j \in J_{1}} \lambda_{i} a_{j s} \mathbf{x}_{s} \\
= & \sum_{j \in J_{2}} \lambda_{j} a_{j 1} \mathbf{x}_{1}+\cdots+\sum_{j \in J_{2}} \lambda_{j} a_{j s} \mathbf{x}_{s} .
\end{aligned}
$$

Since $\mathbf{x}_{1}, \mathbf{x}_{2}, \cdots, \mathbf{x}_{s}$ are strong linearly independent, then $\sum_{j \in J_{1}} \lambda_{j} a_{j k}=\sum_{j \in J_{2}} \lambda_{j} a_{j k}$ with $k \in$ $\underline{s}$. Let $\mathbf{c}_{1}, \mathbf{c}_{2}, \cdots, \mathbf{c}_{s}$ be column-vectors of $A$. Then it is obvious that $\sum_{j \in J_{1}} \lambda_{j} \mathbf{c}_{j}=\sum_{j \in J_{2}} \lambda_{j} \mathbf{c}_{j}$, i.e., $\left\{\mathbf{c}_{1}, \mathbf{c}_{2}, \cdots, \mathbf{c}_{s}\right\}$ is linearly dependent or semilinearly dependent. Thus by Lemma 3.3, we have $\operatorname{det}(A) \equiv 0$, i.e. $\operatorname{det}_{1}(A)=\operatorname{det}_{2}(A)$. In the other hand, by Lemma 3.2 and Definition 3.3 we have

$$
\begin{aligned}
& \operatorname{det}_{1}(A B)+\operatorname{det}_{1}(A) \operatorname{det}_{2}(B)+\operatorname{det}_{2}(A) \operatorname{det}_{1}(B) \\
= & \operatorname{det}_{2}(A B)+\operatorname{det}_{1}(A) \operatorname{det}_{1}(B)+\operatorname{det}_{2}(A) \operatorname{det}_{2}(B) \\
= & \operatorname{det}_{2}(A B)+\operatorname{det}_{2}(A) \operatorname{det}_{1}(B)+\operatorname{det}_{1}(A) \operatorname{det}_{2}(B) .
\end{aligned}
$$

By $K^{+}(L)=L$, it is clear that $\operatorname{det}_{1}(A B)=$ $\operatorname{det}_{2}(A B)$, i.e. $\operatorname{det}(A B) \equiv 0$. But by Eq.(2), Lemma 3.1 we have $A B=I_{s}$, i.e. $\operatorname{det}(A B) \not \equiv 0$, a contradiction. In the second case, there exists $\left|J_{i}\right|=1, i \in\{1,2\}$ such that if $j \in J_{i}$, then $\lambda_{j} \notin U(L)$, say $\left|J_{1}\right|=1$. Then in similar to the proof of the first case, we can prove that this case dose not exist. Therefore by the discuss as above, we know that $\left\{\mathbf{y}_{1}, \mathbf{y}_{2}, \cdots, \mathbf{y}_{s}\right\}$ is strong linearly independent.

By Theorem 3.1, we have the following statement

Corollary 3.1 In a cancellative, yoked and entire semiring $\mathcal{L}$, let $\mathcal{W}=\left\langle\mathbf{x}_{1}, \mathbf{x}_{2}, \cdots, \mathbf{x}_{s}\right\rangle$ with $\left\{\mathbf{x}_{1}, \mathbf{x}_{2}, \cdots, \mathbf{x}_{s}\right\}$ a set of semi-linearly dependent vectors in $\mathcal{V}_{n}$. If $\left\{\mathbf{y}_{1}, \mathbf{y}_{2}, \cdots, \mathbf{y}_{p}\right\}$ is a basis of $\mathcal{W}$, then $\mathbf{y}_{1}, \mathbf{y}_{2}, \cdots, \mathbf{y}_{p}$ are also semi-linearly dependent.

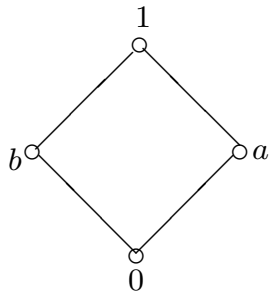

Fig. 1

Note that in Theorem 3.1 the condition of semiring $\mathcal{L}$ is cancellative, yoked and entire can not be deleted generally. For instance, over semiring as Fig. 1, it is obvious that $\mathcal{L}$ is not cancellative, yoked and entire. Then it is easy to see that in $\mathcal{V}_{2},\left\{\left(\begin{array}{l}1 \\ 0\end{array}\right),\left(\begin{array}{l}0 \\ 1\end{array}\right)\right\}$ and $\left\{\left(\begin{array}{l}a \\ 0\end{array}\right),\left(\begin{array}{l}b \\ 0\end{array}\right),\left(\begin{array}{l}0 \\ a\end{array}\right)\right.$, $\left.\left(\begin{array}{l}0 \\ b\end{array}\right)\right\}$ are equivalent and linearly independent. However, $\left\{\left(\begin{array}{l}1 \\ 0\end{array}\right),\left(\begin{array}{l}0 \\ 1\end{array}\right)\right\}$ is a set of strong linearly independent, but $\left\{\left(\begin{array}{l}a \\ 0\end{array}\right),\left(\begin{array}{l}b \\ 0\end{array}\right),\left(\begin{array}{l}0 \\ a\end{array}\right),\left(\begin{array}{l}0 \\ b\end{array}\right)\right\}$ is semi-linearly dependent.

By Theorem 3.1, we also have

Corollary 3.2 In an $\mathcal{L}$-semilinear space $\mathcal{V}_{n}$, if semiring $\mathcal{L}$ is cancellative, yoked and entire, then each basis has the same number of elements.

Lemma 3.4 (Tan [18]) Let $A, B \in M_{n}(L)$. If $A$ is invertible, then $\operatorname{det}(A B)=\operatorname{det}(A) \cdot \operatorname{det}(B)$ and $\operatorname{det}(B A)=\operatorname{det}(B) \cdot \operatorname{det}(A)$.

Lemma 3.5 (Tan [18]) Let $A, B \in M_{n}(L)$. If $A B=I_{n}$, then

(1) $a_{i j} a_{i k}=a_{j i} a_{k i}=b_{i j} b_{i k}=b_{j i} b_{k i}=0$ for any $i, j, k \in \underline{n}$ with $j \neq k$;

(2) $\left(\sum_{k \in \underline{n}} a_{i k}\right)\left(\sum_{l \in \underline{n}} b_{l i}\right)=\left(\sum_{k \in \underline{n}} a_{k j}\right)\left(\sum_{l \in \underline{n}} b_{j l}\right)=$ 1 for any $i, j \in \underline{n}$.

Theorem 3.2 Let $A \in M_{n}(L)$. If $\mathcal{L}$ is entire, then $A$ is invertible if and only if there exists a permutation matrix $P \in M_{n}(L)$ such that $P A$ is an invertible diagonal matrix.

Proof. Suppose that $A$ is invertible. Then there exists a matrix $B$ in $M_{n}(L)$ such that $A B=I_{n}$. From Lemma 3.4, we have $\operatorname{det}(A) \in U(L)$, thus every column of $A$ has a nonzero element and every row of $A$ has a nonzero element. By Lemma 3.5, every column and every row of $A$ has exact one invertible and the others are zeros. Thus there exists a permutation matrix $P \in M_{n}(L)$ such that $P A$ is an invertible diagonal matrix. The converse part directly comes from the hypothesis. 
Lemma 3.6 (Shu and Wang [15]) In semilinear space $\mathcal{V}_{n}$, each basis has the same number of elements if and only if every vector can be uniquely represented by a linear combination of a basis.

Theorem 3.3 In a cancellative, yoked and entire semiring $\mathcal{L}$, let $\mathcal{W}=\left\langle\mathbf{x}_{1}, \mathbf{x}_{2}, \cdots, \mathbf{x}_{s}\right\rangle$ with $\left\{\mathbf{x}_{1}, \mathbf{x}_{2}, \cdots, \mathbf{x}_{s}\right\}$ a set of strong linearly independent vectors in $\mathcal{V}_{n}$. If $\mathbf{y}_{1}, \mathbf{y}_{2}, \cdots, \mathbf{y}_{p} \in \mathcal{W}$, then the follow statements are equivalent:

(1) $\left\{\mathbf{y}_{1}, \mathbf{y}_{2}, \cdots, \mathbf{y}_{p}\right\}$ is a basis of $\mathcal{W}$;

(2) There exists an invertible generalized diagonal matrix $A \in M_{n}(L)$ such that $\left(\mathbf{y}_{1}, \mathbf{y}_{2}, \cdots, \mathbf{y}_{p}\right)=$ $\left(\mathbf{x}_{1}, \mathbf{x}_{2}, \cdots, \mathbf{x}_{s}\right) A$ and $p=s$.

Proof. By Lemma 3.1, it is clear that $s=p$. Let $\mathbf{y}_{i}=\sum_{j=1}^{s} a_{i j} \mathbf{x}_{j}$ with $a_{i j} \in L$ for every $i \in \underline{s}$. Thus

$$
\left(\mathbf{y}_{1}, \mathbf{y}_{2}, \cdots, \mathbf{y}_{s}\right)=\left(\mathbf{x}_{1}, \mathbf{x}_{2}, \cdots, \mathbf{x}_{s}\right) A
$$

with

$$
A=\left(\begin{array}{ccc}
a_{11} & \cdots & a_{s 1} \\
\cdots & \cdots & \cdots \\
a_{1 s} & \cdots & a_{s s}
\end{array}\right)
$$

In a similar way, we can let

$$
\left(\mathbf{x}_{1}, \mathbf{x}_{2}, \cdots, \mathbf{x}_{s}\right)=\left(\mathbf{y}_{1}, \mathbf{y}_{2}, \cdots, \mathbf{y}_{s}\right) B
$$

with

$$
B=\left(\begin{array}{ccc}
b_{11} & \cdots & b_{s 1} \\
\cdots & \cdots & \cdots \\
b_{1 s} & \cdots & b_{s s}
\end{array}\right), b_{j i} \in L, i, j \in \underline{s}
$$

since $\left\{\mathbf{x}_{1}, \mathbf{x}_{2}, \cdots, \mathbf{x}_{s}\right\}$ is a basis of $\mathcal{W}$ by Definition 2.6. Therefore,

$$
\begin{aligned}
& \left(\mathbf{x}_{1}, \mathbf{x}_{2}, \cdots, \mathbf{x}_{s}\right) \\
= & \left(\mathbf{x}_{1}, \mathbf{x}_{2}, \cdots, \mathbf{x}_{s}\right) A B \\
= & \left(\mathbf{x}_{1}, \mathbf{x}_{2}, \cdots, \mathbf{x}_{s}\right) I_{s} .
\end{aligned}
$$

By Lemmas 3.1 and 3.6, $A B=I_{s}$.

\section{Generalized Kronecker- Capelli theorem}

In this section, we shall prove that the KroneckerCapelli theorem for a matrix equation is valid over a commutative zerosumfree semiring.

Definition 4.1 (Shu and Wang [16]) Let $A \in$ $M_{n \times m}(L)$. We call the dimension (if it exists) of column (resp. row) space of $A$ the column (resp. row) rank of $A$, denoted by $r_{c}(A)$ (resp. $\left.r_{r}(A)\right)$. If $r_{c}(A)=r_{r}(A)$, then we say that the rank of $A$, written $r(A)$, is $r_{c}(A)$ or $r_{r}(A)$.

Let $A=\left(a_{i j}\right) \in M_{n \times m}(L)$ and $\mathbf{b}=$ $\left(b_{1}, b_{2}, \cdots, b_{n}\right)^{T} \in M_{n \times 1}(L)$. Consider the following system of equations

$$
\left\{\begin{array}{l}
a_{11} x_{1}+\cdots+a_{1 n} x_{n}=b_{1}, \\
a_{21} x_{1}+\cdots+a_{2 n} x_{n}=b_{2}, \\
\cdots \\
a_{n 1} x_{1}+\cdots+a_{n n} x_{n}=b_{n}
\end{array}\right.
$$

with respect to an unknown vector $\mathbf{x}=$ $\left(x_{1}, x_{2}, \cdots, x_{n}\right)^{T} \in \mathcal{V}_{n}$. Denote the column vectors of $A$ by $\mathbf{a}_{1}, \mathbf{a}_{2}, \cdots, \mathbf{a}_{n}$. Obviously, they are elements of $\mathcal{V}_{n}$.

Lemma 4.1 In a cancellative, yoked and entire semiring $\mathcal{L}$, vectors $\mathbf{a}_{1}, \cdots, \mathbf{a}_{n+1} \in V_{n}(L)$ are either linearly dependent or semi-linearly dependent.

The following theorem is the Kronecker-Capelli theorem for system (4) over a commutative zerosumfree semiring.

Theorem 4.1 In a cancellative, yoked and entire semiring $\mathcal{L}$, let $\mathbf{a}_{1}, \mathbf{a}_{2}, \cdots, \mathbf{a}_{n}$ be strong linearly independent. For every $r \in L$, if $\operatorname{det}(A) \neq \operatorname{rdet}\left(D_{i}\right)$ with $i \in \underline{n}$, then system (4) is solvable if and only if $r(A)=r(A \mathbf{b})$, where the matrix $A \mathbf{b}$ is equal to $A$ extended by $\mathbf{b}$ as the last column and $D_{i}$ is the matrix formed by replacing the $i$-th column of $A$ by the column vector $\mathbf{b}$. Moreover, if system (4) is solvable, then it has a unique solution.

Proof. If system (4) is solvable, then it is clear that $r_{c}(A)=r_{c}(A) \mathbf{b}=r_{r}(A)=r_{r}(A) \mathbf{b}=n$, i.e., $r(A)=r(A \mathbf{b})$. So we just need to prove the sufficiency. If $r(A)=r(A \mathbf{b})$, then by Lemma 4.1 and Definition 4.1 we have that vectors $\mathbf{a}_{1}, \mathbf{a}_{2}, \cdots, \mathbf{a}_{n}, \mathbf{b}$ are linearly dependent. If $\mathbf{b}$ can be represented by a linear combination of $\mathbf{a}_{1}, \mathbf{a}_{2}, \cdots, \mathbf{a}_{n}$, then system (4) is solvable. If there exists $i \in \underline{n}$ such that $\mathbf{a}_{i}$ can be represented by a linear combination of $\mathbf{a}_{1}, \cdots, \mathbf{a}_{i-1}, \mathbf{a}_{i+1} \cdots, \mathbf{a}_{n}, \mathbf{b}$. Say $\mathbf{a}_{1}=k \mathbf{b}+$ $\sum_{j \neq 1, j \in \underline{n}} k_{j} \mathbf{a}_{j}$ with $k, k_{j} \in L, j \neq 1, j \in \underline{n}$. Then we have

$$
\begin{aligned}
\operatorname{det}(A) & =\operatorname{det}\left(k \mathbf{b}+\sum_{j \neq 1, j \in \underline{n}} k_{j} \mathbf{a}_{j}, \mathbf{a}_{2}, \cdots, \mathbf{a}_{n}\right) \\
& =\operatorname{kdet}\left(\mathbf{b}, \mathbf{a}_{2}, \cdots, \mathbf{a}_{n}\right) \\
& +\sum_{j \neq 1, j \in \underline{n}} k_{j} \operatorname{det}\left(\mathbf{a}_{j}, \mathbf{a}_{2}, \cdots, \mathbf{a}_{j}, \cdots, \mathbf{a}_{n}\right) .
\end{aligned}
$$

Therefore we can prove that

$$
\begin{aligned}
& \operatorname{det}_{1}(A)+k \operatorname{det}_{2}\left(D_{1}\right)+\sum_{j \neq 1, j \in \underline{n}} k_{j} \operatorname{det}_{2}\left(D_{j}\right) \\
= & \operatorname{det}_{1}(A)+\operatorname{kdet}_{1}\left(D_{1}\right)+\sum_{j \neq 1, j \in \underline{n}} k_{j} \operatorname{det}_{1}\left(D_{j}\right)
\end{aligned}
$$

and $\sum_{j \neq 1, j \in \underline{n}} k_{j} \operatorname{det}_{1}\left(D_{j}\right)=\sum_{j \neq 1, j \in \underline{n}} k_{j} \operatorname{det}_{2}\left(D_{j}\right)$. Thus $\operatorname{det}(A)=k \operatorname{det}\left(D_{1}\right)$, a contradiction. Therefore if $r(A)=r(A \mathbf{b})$ then system (4) is solvable. By Lemma 3.1, we also know that system (4) has a unique solution.

\section{Conclusions}

In this contribution, we have shown some necessary and sufficient conditions that a set of vectors is a basis of an $\mathcal{L}$-semilinear subspace which is generated by strong linearly independent vectors 
and have proven that the analog of the KroneckerCapelli theorem is valid in an $\mathcal{L}$-semilinear vector space. It is worth to point out that we just discuss the $\mathcal{L}$-semilinear subspace which is generated by strong linearly independent vectors. Therefore, it remains open whether there are other conditions under which the similar results hold.

\section{Acknowledgements}

This was supported by National Natural Science Foundation of China (Nos. 11401410, 11171242 and 11201325).

\section{References}

[1] F. L. Baccelli, G. Cohen, G. J. Olsder and J. P. Quadrat, Synchronization and Linearity, Chichester, J. Wiley and Sons, New York, 1992.

[2] P. Butkovič, Max-algebra: the linear algebra of combinatorics? Linear Algebra and its Applications 367:313-335, 2003.

[3] Z. Q. Cao, K. H. Kim, F. W. Roush, Incline Algebra and Applications, John Wiley, New York, 1984.

[4] K. Cechlárová, J. Plávka, Linear independence in bottleneck algebras, Fuzzy Sets and Systems 77: 337-348, 1996.

[5] R. A. Cuninghame-Green, Minimax algebra, Lecture Notes in Economics and Mathematical Systems, Vol. 166, Springer, Berlin, 1979.

[6] R. A. Cuninghame-Green, P. Butkovič, Bases in max-algebra, Linear Algebra and its Applications 389: 107-120, 2004.

[7] A. Di Nola, A. Lettieri, I. Perfilieva, V. Novák, Algebraic analysis of fuzzy systems, Fuzzy Sets and Systems 158: 1-22, 2007.

[8] J. S. Golan, Semirings and Their Applications, Kluwer Academic Publishers, Dordrecht/Boston/London, 1999.

[9] M. Gondran, M. Minoux, Linear algebra in dioïds: a survey of recent results, Ann. Discrete Mathematics 19: 147-164, 1984.

[10] K. H. Kim, F. W. Roush, Generalized fuzzy matrices, Fuzzy Sets and Systems 4: 293-315, 1980.

[11] I. Perfilieva, Semi-linear spaces, in: H. Noguchi, H. Ishii, et al., editor, Proc. of Seventh Czech-Japanese Seminar on Data Analysis and Decision Making under Uncertainty, pages 127-130, Hyogo, Japan, 2004.

[12] I. Perfilieva, J. Kupka, Kronecker-capelli theorem in semilinear spaces, In D. Ruan, T. R. Li, Y. Xu, G. Q. Chen and E. E. Kerre, Computational Intelligence: Foundations and Applications, World Scientific, pp. 43-51, Emei, Chengdu, China, 2010.

[13] Phillip L. Poplin, Robert E. Hartwig, Determinantal Identities over Commutative semirings,
Linear Algebra and its Applications 387: 99-132, 2007.

[14] O. A. Pshenitsyna, Factor and term ranks for matrix union over semirings, Journal of Mathematical Sciences 5: 3384-3399,2006.

[15] Qian-yu Shu, Xue-ping Wang, Bases in semilinear spaces over zerosumfree semirings, Linear Algebra and its Applications 435: 2681-2692, 2011.

[16] Qian-yu Shu, Xue-ping Wang, Standard orthogonal vectors in semilinear spaces and their applications, Linear Algebra and its Applications 437: 2733-2754, 2012.

[17] Qian-yu Shu, Xue-ping Wang, Dimensions of L-semilinear spaces over zerosumfree semiring, 2013 Joint IFSA World Congress and NAFIPS Annual Meeting (IFSA/NAFIPS), pages 35-40.

[18] Y. J. Tan, On invertible matrices over antirings, Linear Algebra and its Applications 423: 428-444, 2007.

[19] Xue-ping Wang, Qian-yu Shu, Bideterminant and rank of matrix, Soft Comfuting,18: 729-742, 2014.

[20] Shan Zhao, Xue-ping Wang, Invertible matrices and semilinear spaces over commutative semirings, Information Sciences 180: 5115-5124, 2010.

[21] Shan Zhao, Xue-ping Wang, Bases in semilinear spaces over join-semirings, Fuzzy Sets and Systems 182: 93-100, 2011.

[22] U. Zimmermann, Linear and combinatorial optimization in ordered algebrac structures, North Holland, 1981. 